\title{
PMMA wettability caused by ultraviolet radiation
}

\author{
Yu. Dekhtyar ${ }^{1}$, L. Lancere ${ }^{1}$, N. Polyaka ${ }^{1}$, A. Sudnikovich ${ }^{1}$, F. Tyulkin ${ }^{1}$, \\ V. Valters ${ }^{2}$ \\ ${ }^{1}$ Riga Technical University, 1 Kalku str Riga LV1658, Latvia \\ ${ }^{2}$ Valter's Prosthetic Laboratory, 16 Ledurgas str. Riga LV1034, Latvia
}

\section{Summary}

The article is targeted to explore ultraviolet radiation (UV) influence on PMMA for eye prostheses. UV being the Sun light component and could effect PMMA surface that in turn contributes interaction with tear. PMMA wettability was powered by UV.

\section{Introduction}

PMMA is in use for eye prostheses that during exploitation become under Sun light, UV being the most danger its component. UV could influence the PMMA surface wettability that gives uncomfortable condition for the patients.

$n$ focus, (b)

Sun UV consists of three bands: UVA (315-400 nm), UVB(280-315 nm), UVC (100-280 nm). Just UVA and UVB reach the Earth surface

There are experiments on UVC (192-248 nm) influence on PMMA, particularly on optical properties (refraction), reconstruction of chemical bonds [1-3]. There is a deficit of knowledge on the wettability properties influenced by UVA, UVB.

Therefore the research is focused on the $280-400 \mathrm{~nm}$ range that could affect PMMA surface.

\section{Methods and materials}

The specimens were prepared in a manner as it is in use to fabricate eye prostheses. First the PMMA was polymerized. Further mechanical treatment was applied (slipping and polishing). Finally $96 \%$ ethanol was employed to wash/clean the specimens. The specimens were radiated by the $\mathrm{Hg}$-Xe tube light having an intensity $3.5 \mathrm{~W} / \mathrm{cm}^{2}$. The specimens were positioned at 1.5 $\mathrm{m}$ distance from the lamp, the light spot was equal to $5 \mathrm{~mm}$ and the temperature of the specimens was $+20^{\circ} \mathrm{C} \pm 2^{\circ} \mathrm{C}$. Wettability was tested using Axisymmetric Drop Shape Analysis-Profile (ADSA-P) method.

Wettablity in generally could be affected by both electrical charging of the surface due to photoelectron emission and reconstruction of the chemical couples.

The photoelectron spectrometer was employed to detect the electron work function $(\varphi)$ that alteration is directly connected to the increment of the surface charge. To indicate possible reconstruction of the chemical couples optical absorption was measured. To verify possible surface morphological as well as electrical reconstructions at the nano/micro scale the specimens were scanned using Solver P-47 Pro atomic force microscope, semi contact topography and Kelvin probe methods were in use. 


\section{Results}

Both increment $(\Delta \alpha)$ of the contact angle (Fig. 1) and $\varphi$ were resulted by radiation and demonstrated a non linear behavior characterized with the maximum at 150 minutes of exposure.

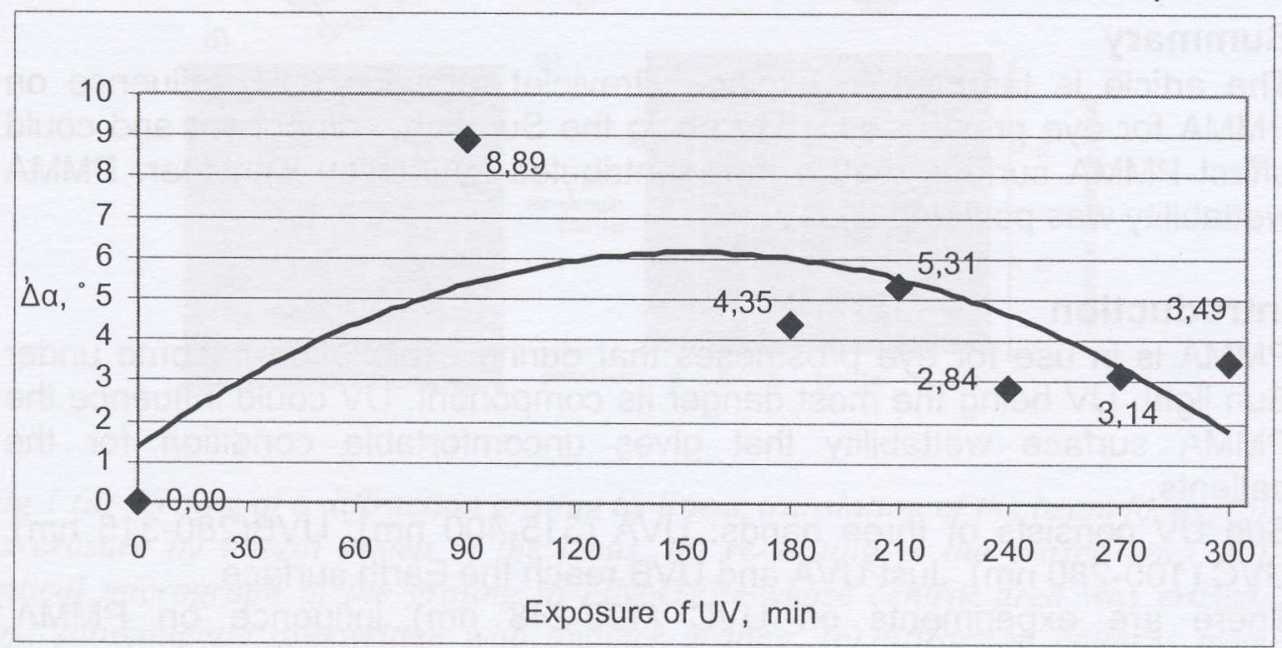

Fig.1. Contact angle increment due to radiation exposure.

The absorbance $(A)$ spectra were characterized with the minimum of the derivation $\mathrm{dA} / \mathrm{d} \lambda$ ( $\lambda$ - the wave length) at $\sim 380 \mathrm{~nm}$. The integral $S=\int_{\lambda} \frac{d A}{d \lambda} d \lambda$ correlated with the exposure of radiation.

The morphology of the surface was not influenced by radiation. However the surface electrical potential depended on exposure under light: autocorrelation function length was powered by radiation.

\section{Conclusion}

Wettability of PMMA could be engineered by UV that affects molecular structure and surface electrical charge.

\section{References.}

[1] Photoinduced Change in Refractive Index of Functional Polymers or Ophthalmic Applications, Dissertation ,Jens Kristian Träger, Marburg/ Lahn, p.35, 172, 2009.

[2] Ranby J, Rabek JF. Photodegradation, Photo-oxidation and Photostabilization of Polymers. London: John Wiley, 156, (1975)

[3] Caykara T., Guven O., UV degradation of poly(methyl methacrylate) and its vinyltriethoxysilane containing copolymers, Department of Chemistry, Faculty of Art and Science, Gazi University, Hacettepe University, Ankara, Turkey, 225229, (1999). 